\title{
Terapia Comunitária Integrativa como estratégia de enfrentamento às drogas entre internos de comunidades terapêuticas: pesquisa documental
}

\author{
Alisséia Guimarães Lemes ${ }^{1}$ \\ Vagner Ferreira do Nascimento ${ }^{2}$ \\ Elias Marcelino da Rocha ${ }^{3}$ \\ Adaene Alves Machado de Moura ${ }^{4}$ \\ Margarita Antonia Villar Luis ${ }^{5}$ \\ Jaqueline Queiroz de Macedo ${ }^{6}$
}

\begin{abstract}
Neste estudo, objetivou-se analisar os registros de fichas de rodas de Terapia Comunitária Integrativa, quanto aos problemas elencados e às estratégias enunciadas para enfrentamento. Trata-se de pesquisa retrospectiva, documental, realizada em município do Estado do Mato Grosso, cujos dados foram coletados por meio de 22 fichas de registro de rodas realizadas em duas comunidades terapêuticas masculinas, no ano de 2015. Na análise categorial dos dados, foram evidenciados os problemas (sentimentos de perdas geradas pelo processo de dependência de drogas, ansiedade e medo da recaída) mais discutidos, bem como apontouse a representatividade da Terapia Comunitária Integrativa como estratégia de apoio no auxílio para o enfrentamento por meio da confiança e do resgate de vínculos, bem como no estímulo, mediante conceitos como esperança e fé, ao suporte emocional.

Descritores: Apoio Social; Comunidade Terapêutica; Transtornos Relacionados ao Uso de Substâncias; Participação Comunitária.
\end{abstract}

\footnotetext{
${ }^{1}$ MSc, Professor Assistente, Universidade Federal de Mato Grosso, Barra do Garças, MT, Brasil.

${ }^{2}$ Doutorando, Centro Universitário São Camilo, São Paulo, SP, Brasil. Professor Assistente, Universidade Estadual de Mato Grosso, Tangará da Serra, MT, Brasil.

${ }^{3}$ MSc, Professor Assistente, Universidade Federal de Mato Grosso, Pontal do Araguaia, MT, Brasil.

${ }^{4}$ Aluna do curso de graduação em Enfermagem, Universidade Federal de Mato Grosso, Barra do Garças, MT, Brasil.

${ }^{5} \mathrm{PhD}$, Professor Titular, Escola de Enfermagem de Ribeirão Preto, Universidade de São Paulo, Centro Colaborador da OPAS/OMS para o Desenvolvimento da Pesquisa em Enfermagem, Ribeirão Preto, SP, Brasil.

${ }^{6} \mathrm{PhD}$, Professor, Universidade Federal da Paraíba, João Pessoa, PB, Brasil.
} 


\title{
Integrative Community Therapy as a strategy for coping with drug among inmates in therapeutic communities: documentary research
}

\begin{abstract}
The aim of this study was to analyze the records from rounds of Integrative Community Therapy regarding the problems listed and the strategies named for dealing with them. This is retrospective documentary research conducted in Barra do Garças/MT, the data for which were taken from 22 records of therapy conducted in two male therapeutic communities in 2015. Content analysis of the data revealed the problems most commonly discussed (feelings of loss stemming from the processes of drug addiction, and Anxiety and fear of relapse), as well as showing the representativeness of $\mathrm{TCl}$ as a support strategy, helping the inmates deal with their problems through trust and renewed links and providing emotional support through hope and faith
\end{abstract}

Descriptors: Social Support; Therapeutic Community; Substance-Related Disorders; Community Participation.

\section{Terapia Comunitaria Integrativa como estrategia de afrontamiento de} las drogas entre internos de comunidades terapéuticas: investigación documental

\begin{abstract}
Este estudio se realizó con el objetivo de analizar los registros de las ruedas de Terapia Comunitaria Integrativa en cuanto a los problemas enumerados y estrategias para el afrontamiento. Se trata de una investigación retrospectiva, documental realizada en ciudad de Mato Grosso, cujos datas furon recolectados por medio de 22 fichas de registro de ruedas realizadas en dos comunidades terapéuticas masculinas en el año 2015. En el análisis de categorías de los datos evidenció los seguintes problemas (sentimiento de pérdidas generadas por el proceso de dependencia de las drogas, ansiedad y miedo a la recaída), mas discusiones revelaran como el $\mathrm{TCl}$ es una estrategia de apoyo, la cual auxilia el enfrentamiento por medio de la confianza y rescate de vínculos lo que proporciona, estimulo, el apoyo emocional a través de la esperanza y la fe.
\end{abstract}

Descriptores: Apoyo Social; Comunidad Terapéutica; Trastornos Relacionados con Sustancias; Participación Comunitaria.

\section{Introdução}

Os agravos decorrentes da dependência química têm provocado problemas cada vez mais preocupantes no Brasil. Consequentemente, aumentou-se a busca por ações preventivas, de reabilitação e de reinserção desse sujeito na sociedade $^{(1-2)}$. Embora, no país, exista a Política
Nacional sobre Drogas (PNSD), que garante o tratamento baseado em acompanhamento integral do usuário de drogas, ampliado por meio de ações de redução de danos e de reinserção, suas medidas não têm sido eficazes no sentido de reduzir o consumo entre as pessoas dediversas faixas etárias, classes e gêneros, tampouco frear a violência em face do tráfico e consumo de drogas ${ }^{(3-4)}$. 
No Brasil, entre as drogas mais consumidas estão o álcool, como droga lícita, e a maconha, como ilícita. Quanto ao consumo por gênero, prevalece entre os homens o uso de álcool, tabaco, maconha, cocaína e crack e, entre as mulheres, estimulantes, benzodiazepínicos, orexígenos e opiáceos ${ }^{(5)}$. Nesse sentido, no II Levantamento Domiciliar sobre o Uso de Drogas Psicotrópicas, realizado em 2004, no Brasil, apontou-se a maconha como a droga ilícita mais consumida na Região Centro-Oeste $(7,8 \%$ dos entrevistados), sendo seu consumo três vezes maior entre homens, com destaque para a faixa etária de 25 a 34 anos $^{(6)}$. Já no último levantamento realizado em 2010, revelou-se que, quanto ao uso ao longo da vida, houve aumento no consumo da maconha, comparado ao levantamento de 2004 em Campo Grande (de 3,7 a 4,5\%) e Cuiabá (de 3,7 a 4,8\%), e de cocaína em Brasília (de 1,8 a 4,1\%) e Cuiabá (de $1,4$ a $3 \%)^{(3)}$.

Em estudos, aponta-se que o consumo de drogas causa consequências devastadoras na vida do usuário, envolvendo estrutura familiar, vida social, condição de saúde, compromisso com os estudos e, consequentemente, vida profissional(1-2).

Pensando naqueles que fazem uso abusivo de drogas e se encontram em situação de dependência, várias formas de atenção à saúde têm sido organizadas a fim de atender essa comunidade, como, por exemplo, a possibilidade do tratamento realizado mediante internação em hospital geral para desintoxicação, atendimento em Centros de Atenção Psicossocial para Álcool e Drogas (CAPS ad) e atendimento em Comunidades Terapêuticas $(\mathrm{CT})^{(5)}$.

Entre essas estratégias, o tratamento deve ocorrer por meio de "abordagem interdisciplinar a partir de intervenções psicoterápicas e sociais, com vistas à reabilitação e reinserção social dos dependentes químicos" $(7)$.

$\mathrm{Na}$ proposta de oferecer ao dependente de drogas formas de cuidado complementares no processo de reabilitação, tem sido utilizada a Terapia Comunitária Integrativa ( $\mathrm{TCl}$ ). A $\mathrm{TCl}$ foi criada em 1987, pelo psiquiatra e antropólogo Dr. Adalberto de Paula Barreto, docente do Departamento de Saúde Comunitária da Faculdade de Medicina da Universidade Federal do Ceará (Famed/UFC) $)^{(4,8)}$.

Essa abordagem complementar encontra-se inserida no contexto do Sistema Único de Saúde (SUS), sendo utilizada como método de cuidado leve, para prevenir o surgimento de doenças ocasionadas por situações estressantes e conflituosas vivenciadas no dia a dia das pessoas ${ }^{(4)}$.
A TCl "baseia-se na troca de experiências e vivências da comunidade, a partir da escuta das histórias de vida e da superação do sofrimento, onde todos são corresponsáveis na busca de soluções e superação dos desafios do cotidiano"(9). Esse método de realização ocorre em roda e compreende cinco etapas: acolhimento, escolha do tema, contextualização, problematização e encerramento ${ }^{(8)}$.

A TCl foi inserida em Cuiabá, MT, Brasil, por meio do Grupo de Pesquisa Enfermagem Saúde e Cidadania (GPESC) e do Programa de Educação Tutorial Conexões de Saberes (PET Conexões de Saberes) da Universidade Federal de Mato Grosso (UFMT) de Cuiabá, desde 2009. Já em 2015, a TCI foi expandida para o interior do Estado mediante ações do projeto de extensão Terapia Comunitária Integrativa: entre nessa roda, do Campus Universitário do Araguaia (CUA-UFTM).

A TCl mostra-se importante para alguns grupos, como os compostos de dependentes químicos em processo de recuperação, possibilitando reconhecer suas vulnerabilidades nas relações, experiências de vida e dependência ${ }^{(10)}$. Em face do exposto neste estudo, teve-se como objetivo analisar os registros das fichas quanto aos problemas elencados nas rodas de $\mathrm{TCl}$, bem como as estratégias enunciadas para enfrentamento.

\section{Metodologia}

Trata-se de pesquisa retrospectiva, do tipo documental, de natureza qualitativa, realizada na Universidade Federal de Mato Grosso (UFMT), Campus Universitário do Araguaia (CUA), em Barra do Garças, MT, Brasil. Os documentos de registro fazem parte do acervo do grupo de extensão Terapia Comunitária Integrativa: entre nessa roda, da UFMT/ CUA, para tanto, teve-se autorização da coordenação do projeto de extensão para a utilização dos documentos.

A fonte de coleta de dados deste estudo foram documentos originais de registro da $\mathrm{TCl}$, denominados Ficha de organização das informações das rodas de $\mathrm{TCl}$, nos quais há informações como número de participantes nas rodas, principais problemas vivenciados, estratégias sugeridas como forma de enfrentamento e depoimentos espontâneos sobre a terapia vivenciada. No caso deste estudo, as fichas coletadas foram registradas por três enfermeiros terapeutas do município, no período de fevereiro a dezembro de 2015. 
Desse modo, foram consultadas 55 fichas, das quais apenas $40 \%$ atenderam os critérios de inclusão: fichas de rodas de $\mathrm{TCl}$, realizadas com internos de CTs, com preenchimento adequado de todos os campos de registro das rodas de TCl. Sendo assim, as 22 fichas selecionadas para este estudo decorreram de rodas de $\mathrm{TCl}$ realizadas em duas $\mathrm{CTS}$ do município, instituições essas de natureza religiosa, com capacidade para 30 homens com idade igual ou superior a 18 anos, usuários de álcool, crack e outras drogas, em situação de dependência química, independentemente do fato de terem ou não estado em situação de rua.

O acesso de usuários às CTs ocorre por meio de demanda espontânea, ordem judicial e encaminhamento informal da rede de atenção psicossocial da região, sendo que a internação pode variar entre seis meses (álcool) e nove meses (crack e outras drogas). A assistência médica aos usuários das CTs é realizada pelo CAPS ad, mediante parceria com a secretaria municipal de saúde. As demais atividades de promoção da saúde, reabilitação e reinserção dos usuários são realizadas por profissionais voluntários ou acadêmicos de cursos da saúde, vinculados a práticas e projetos de pesquisa e extensão de faculdades públicas ou privadas.

Os dados foram analisados de acordo com a análise categorial(11) e os achados, organizados em categorias analíticas, segundo os temas mais enunciados pelos participantes.

A pesquisa foi aprovada pelo Comitê de Ética em Pesquisa da UFMT/CUA, sob Protocolo $n^{\circ}$ 515/705, seguindo as observâncias éticas preconizadas na Resolução 466/2012, do Conselho Nacional de Saúde (CNS), a qual dispõe sobre pesquisas em seres humanos no país.

\section{Resultados e discussão}

A amostra foi constituída de 22 fichas de organização das informações da CTI, realizada em duas CTs masculinas, no período de janeiro a dezembro de 2015. A média de participantes nas rodas de TCl foi de 14,63 homens, com faixa etária entre 19 e 68 anos.

\section{A. Problemas evidenciados pelos participantes}

Os problemas vivenciados por homens dependentes químicos em processo de recuperação compõem o quarto e quinto itens da ficha de registro. Durante a TCl, a coleta dessa informação ocorre na fase de escolha do tema da roda, em que todos os participantes são instigados a falar de problemas que estão lhes angustiando.

De acordo com estudos, esses problemas podem estar relacionados ao emocional, ao psicológico, à desestrutura familiar, a problemas sociais e de saúde, gerados na vida do dependente de drogas ${ }^{(4)}$. Neste estudo, os problemas serão apresentados em duas subcategorias: sentimentos de perdas geradas pelo processo da dependência de drogas, a ansiedade e medo da recaída.

\section{A.1 Sentimentos de perdas geradas pelo processo da dependência de drogas}

Os sentimentos de perdas estão relacionados aos prejuízos ocasionados pela dependência de drogas, em todos os aspectos (social, econômico, da saúde e vínculos familiares), e essas perdas podem, em longo prazo, expor o usuário a situação de criminalidade e/ ou mendigação na tentativa de sustentar o vício ${ }^{(12)}$.

Pautando-se nos processos que envolvem perdas, neste estudo foram representados registros referentes à ausência do contato familiar $(27 \%)$, a sentimentos de frustrações $(9 \%)$ e problemas de saúde $(5 \%)$.

Em estudos, aponta-se que o abuso e a dependência de drogas ocasionam danos e frustrações que acometem as várias dimensões do ser humano e, principalmente, da família ${ }^{(13)}$. Tais consequências estão relacionadas a todo o processo de drogadição, envolvendo o indivíduo e a família. A família passa por constantes situações de crise, o que torna as relações entre seus membros ameaçadas, necessitando constantemente que os vínculos sejam refeitos e/ou reorganizados $^{(14)}$. Aponta-se, também, que a família pode contribuir no processo de recuperação do dependente, colaborando, de forma participativa e ativa, no plano de tratamento do usuário, preparandose para acolhê-lo posterirormente ao tratamento ${ }^{(7)}$.

Entre os sentimentos de frustrações revelados pelos registros encontram-se as dificuldades financeiras e o medo da recaída. Sentimentos esses ocasionados pela dependência de drogas. Na perspectiva de reabilitação do usuário, é preciso que ele aprenda a lidar com o processo de frustração, em todos os âmbitos de sua vida (social, econômico, afetivo etc.) ${ }^{(15)}$.

Em estudo qualitativo, realizado no interior do Estado do Mato Grosso, com internos de CTs, também foram revelados sentimentos de frustrações (perda e medo) entre os participantes, corroborando a pesquisa aqui apresentada ${ }^{(10)}$. 
A TCl proporciona o local adequado para que as pessoas possam se comunicar e autoconhecer, elaborando uma rede viva de comunicações gestuais pela qual cada um pode expressar sentimentos, sejam eles de alegria, tristeza, medo, angústia, frustração, fortalecendo-os para reagir face a situações adversas, considerando-se que as rodas de $\mathrm{TCl}$ representam o local em que os sentimentos de frustrações são revistos e trabalhados ${ }^{(8)}$.

Outro problema revelado neste estudo foi a preocupação com a saúde. É importante ressaltar que a saúde física e psíquica do indivíduo é fragilizada pelo uso abusivo de drogas, quando comparada com outras doenças, representando um dos maiores problemas de saúde pública(5).

Assim, durante o processo de dependência de drogas, é comum os usuários adquirirem problemas de saúde devido ao estilo de vida desregrado, assumindo comportamento de risco em busca da manutenção do vício, tais como: alterações psicoemocionais (exemplo: impulsividade, alterações do humor) e doenças agudas e crônicas (ex.: Aids, hepatite, tuberculose, hanseníase, desnutrição, endocardite, enfisema pulmonar). Tais afecções surgem como complicações, que carecem de intervenções dos serviços de saúde, aliadas ao tratamento para a dependência química proposto(16).

\section{A.2 Ansiedade e medo da recaída}

Sentimentos como a ansiedade e o medo são comuns entre os usuários de drogas, principalmente no processo de recuperação da dependência, devido às incertezas e perdas vivenciadas, e agravado pela sensação iminente de recaída.

Neste estudo, a ansiedade foi expressa por meio da angústia, agitação motora e crises de choro em 14\% dos registros, e o medo da recaída, por sentimentos como vergonha, culpa, raiva e desesperança em 14\% dos problemas registrados nas fichas.

Quanto à ansiedade, é sintoma comum entre internos de CTs ou quaisquer outros locais de tratamento, podendo manifestar-se por meio de tristeza, angústia, agitação motora e, também, choro frequente(10).

No caso dos participantes da $\mathrm{TCl}$ analisada neste estudo, o processo de ansiedade possivelmente foi gerado pelo fato de os internos ficarem, na maior parte do tempo, ociosos, longe da família, da rotina estabelecida antes da internação e, até mesmo, pelo processo de abstinência que o tratamento realizado nas CTs estudadas gera na vida do indivíduo.
Aliado a isso há, ainda, o medo da recaída. O processo de recaída refere-se ao padrão de uso anterior ao início da intervenção, porém, todo esse processo está arraigado em sentimentos como vergonha, culpa, raiva, tristeza, frustração e desesperança. Em estudos, afirma-se que, socialmente, os usuários, na sua maioria, quando em processo de recuperação, se comprometem a não mais fazer uso de drogas, porém, sentem-se constantemente ameaçados pela recaída ao saírem do local de internação ${ }^{(17)}$.

Diante desses problemas evidenciados nas duas subcategorias apresentadas acima, é possível afirmar que, com o uso da terapia comunitária, busca-se minimizar os sentimentos e sofrimentos dos participantes, a fim de oportunizar aos usuários momentos de escuta, respeito, expressão dos sentimentos e partilha, a qual pode ser realizada por meio da expressão da fala, do choro e do canto ${ }^{(8,18)}$.

Nesse sentido, ao colocar a situação-problema na roda, o indivíduo oferece ao grupo a possibilidade de reflexão mais ampla, em que estão incluídos os diversos fatores e elementos do contexto do sistema econômico-social, possibilitando a percepção de que a problemática em questão é comum aos demais, e a solução depende, sobretudo, das transformações sociais, com as quais os integrantes podem, individualmente, contribuir no processo de recuperação do outro e, juntos, alcançar melhores resultados ${ }^{(8,19)}$.

O problema, que até então era vivenciado apenas pelo participante de forma individual, passa a ser compartilhado na roda de terapia, onde todos compartilham a dor vivida pelo outro(19). O sofrimento partilhado na roda torna-se, de certo modo, um sentimento comum, humano e comunitário(19).

Nos registros, revelou-se que a $\mathrm{TCl}$ representou local de meditação, verbalização entre as pessoas que tiveram oportunidade para expor seus medos, problemas relatados a partir dos seus sentimentos e angústias, podendo desenvolver relações de troca de experiências, de compartilhamento, de modificação, de relacionamento e busca por alternativas para o seu bem-estar(20). Com isso, confirma-se a importância de os indivíduos encontrarem recursos internos e externos para a superação de suas dificuldades, trocando experiências e percebendo que não estão sozinhos em seu abandono e marginalidade ${ }^{(20)}$.

\section{B. Representatividade da TCI como estratégia de apoio}

A $\mathrm{TCl}$ possibilita às pessoas que se sintam chamadas para expressar seu sofrimento cotidiano, 
o que, por sua vez, concorre para o alívio da dor. É entendida, ainda, como local para partilha, para o alívio do sofrimento mental que aflige muitos dependentes ${ }^{(9,21)}$.

Neste estudo, as estratégias mais registradas foram: confiança $(77 \%)$, resgate de vínculos $(68 \%)$, fé $(55 \%)$, esperança $(50 \%)$, superação $(23 \%)$, empoderamento (23\%) e autoconhecimento (17\%), as quais serão discutidas nas duas subcategorias a seguir.

\section{B.1 Enfrentamento por meio da confiança e resgate de vínculos}

Aponta-se, nos registros, que uma das estratégias de enfrentamento mais citadas foi a confiança, principalmente relacionada ao tratamento da dependência química, sendo manifestada por meio do desejo de superar o vício e pela busca de apoio mútuo entre os integrantes para a superação das dificuldades enfrentadas. A confiança é o sentimento de convicção sobre algo ou alguém necessário para que as pessoas possam se relacionar e manter compromissos. No sentido de utilizar a confiança como estratégia de enfrentamento da dependência, a $\mathrm{TCl}$ apoia-se em um dos seus fundamentos teóricos, a saber, o de instigar no participante o empoderamento pessoal, mediante a socialização de vivências cotidianas expressas pelos demais participantes ${ }^{(22)}$.

Outros termos elencados como estratégias e apontados pelos internos para o enfrentamento foram a superação, o empoderamento e o autoconhecimento que, neste estudo, encontram-se representados como a resiliência. A resiliência é a capacidade inerente ao ser humano de se transformar e de transformar sua realidade, superando ou adaptandose às adversidades do dia a dia, a partir de elementos internos e do ambiente que lhe permitem criar essa capacidade de superação(23).

Em estudos, aponta-se que a $\mathrm{TCl}$ oportuniza um mundo comum de argumentação sobremaneira particular, pois os participantes das rodas de TCl têm a oportunidade de refletir sobre suas vidas, elaborando mudanças em seus cotidianos ${ }^{(18,21,23-24)}$. Além disso, está lançada no contexto de seus participantes, possibilitando a esses reconhecerem sua própria realidade e estimulando-os a novas descobertas e reinvenções, edificadas coletivamente ${ }^{(24)}$.

A TCl contribui, também, como ferramenta em que é valorizado o poder de resiliência dos seus participantes, pois desenvolve atividades em grupo que fortalecem os participantes, individual e coletivamente, em prol da busca de novas estratégias voltadas ao enfrentamento dos problemas $^{(20)}$.
Outra estratégia sugerida pelos participantes, registrada nas fichas e relevante nesta pesquisa foi o resgate dos vínculos, por meio da aproximação da família, da formação de novos vínculos de amizades e da busca de trabalho renumerado. A pobreza de relações sociais consiste em fator nocivo à saúde, que pode ser evidenciada na quebra dos vínculos familiares, sejam nucleares ou não, pois esses vínculos podem estar rodeados por angústia, tristeza, estresse, desgaste e descrença, em decorrência do processo de dependência e das recorrentes recaídas durante o tratamento dos usuários de drogas ${ }^{(21)}$. É importante ressaltar que essa quebra no vínculo, motivada pelo desgaste em relações familiares e sociais, causa no usuário sentimentos de solidão, desânimo e insignificância da vida(12)

A "Terapia Comunitária Integrativa desempenha um papel importante como espaço de construção de uma teia de apoio, uma estratégia de criação e de fortalecimento de vínculos sociais e ferramenta para despertar a capacidade de resiliência, pois, é um espaço onde histórias são contadas, e os participantes se reconhecem nas histórias do outro"(23).

Nesse sentido, a TCl colabora para a formação e/ou fortalecimento de vínculos da comunidade, estratégia importante, pois promove mudanças ao focalizar mais o coletivo, levando ao empoderamento da comunidade em virtude da partilhar de experiências $^{(22)}$

\section{B.2 Estímulo ao suporte emocional}

O suporte emocional foi expresso, neste estudo, principalmente pela esperança e fé, sentimentos esses que estão descritos entre os significados de participação dos internos na finalização das rodas de $\mathrm{TCl}$.

A esperança relaciona-se à expectativa que internos têm de vivenciarem experiências positivas. Nesse sentido, os participantes da TCI são fortalecidos por acreditarem em resultados positivos diante do enfrentamento da dependência de drogas ${ }^{(4)}$.

Observou-se, ainda, nos registros, a expressão da fé relacionada à busca da paz interior para o alívio do sofrimento. É importante ressaltar que as comunidades terapêuticas participantes do estudo são de cunho religioso, cujo ambiente é considerado referência para o sujeito e para a família, pois contribui com o afastamento do indivíduo da vivência conturbada com as drogas, oferecendo espaço de recuperação e apoio espiritual, atendendo às angústias imediatas de usuários e familiares 
que, muitas vezes, não encontram respostas nos serviços públicos ${ }^{(10,25)}$.

O incentivo ao exercício da espiritualidade pode favorecer o processo de reabilitação para abuso e dependência de álcool e/ou outras drogas, de maneira competente tal processo pode estar associado à inclusão de novo estilo de vida no qual vínculos, práticas cotidianas e, até mesmo, a participação em determinada religião são encorajadas, para que os internos consigam afastar-se de situações que propiciem o uso de substâncias psicoativas ${ }^{(26)}$.

Para muitas pessoas a fé têm sido utilizada como instrumento de superação dos problemas. Com a fé, alia-se a aquisição de novos comportamentos de vida capazes de despertar no indivíduo a força interior que pode contribuir no processo de recuperação(12). Porém, Braga e colaboradores ${ }^{(23)}$ reforçam que, além da religião, devem existir outras alternativas (sociais, políticas) em busca de suporte ao dependente químico ${ }^{(23)}$.

Nesse sentido, com os dados aqui apresentados, revela-se que os participantes podem experimentar transformações durante a interação das rodas de TCl, contribuindo com o desenvolvimento de elementos que estimulem a capacidade crítica e reflexiva, bem como o reestabelecimento ou criação de vínculos que geram a compreensão necessária à mudança ${ }^{(8)}$.

\section{Conclusão}

Por meio dos registros, foi possível identificar que os problemas mais evidenciados foram as perdas familiares, frustrações, problemas de saúde, angústia e medo. Por outro lado, os sentimentos positivos relacionaram-se à confiança no resgate de vínculos afetivos e à obtenção de suporte emocional.

Revela-se, com tais registros, que a dinâmica de realização da $\mathrm{TCl}$ instigou os usuários a falarem de seus problemas, auxiliando-os e encorajando-os ao resgate de suas forças e autonomia, proporcionado ambiente favorável ao acolhimento das diversas e singulares circunstâncias de vida, o que pôde auxiliar no processo de enfrentamento dos sofrimentos.

A limitação, neste estudo, refere-se ao fato de se tratar de pesquisa documental, desse modo, os participantes não foram contatados diretamente, o que inviabiliza a identificação das expressões e vivências particulares do grupo.

Para tanto, com os resultados deste estudo e de outros descritos na literatura em contextos similares, aponta-se que a $\mathrm{TCl}$ é efetiva na compreensão do espaço de cuidado coletivo, promovendo saúde e bem-estar aos sujeitos por meio da socialização e partilha de suas histórias, importante ferramenta voltada ao cuidado complementar de pessoas com dependência de drogas.

\section{Referências}

1. Ventura CAA. Drogas lícitas e ilícitas: do direito internacional à legislação brasileira. Rev Eletrôn Enferm. 2011;13(3):560-5.

2. Damas FB. Comunidades Terapêuticas no Brasil: expansão, institucionalização e relevância social. Rev Saúde Pública Santa Catarina. 2013;6(1):50-65.

3. Carlini ELA, Noto AR, Sanchez ZM. VI Levantamento Nacional sobre o Consumo de Drogas Psicotrópicas entre Estudantes do Ensino Fundamental e Médio das Redes Pública e Privada de Ensino nas 27 Capitais Brasileiras. Brasília: Secretaria Nacional de Políticas sobre Drogas-SENAD; 2010. 503 p.

4. Ferreira Filha $M O$, Sá ANP, Rocha IA, Silva VCL, Souto CMRM, Dias MD. Alcoolismo no contexto familiar: estratégias de enfrentamento das idosas usuárias da Terapia Comunitária. Rev RENE. 2012;13(1):26-35.

5. Duarte PCAV, Stempliuk VA, Barroso LP. Relatório brasileiro sobre drogas. Brasília: Secretaria Nacional de Políticas sobre Drogas-SENAD. 2009. 48 p.

6. Carlini ELA, Galduróz JCF, Silva AAB. II Levantamento domiciliar sobre o uso de drogas psicotrópicas no Brasil: estudo envolvendo as 108 maiores cidades do país. São Paulo: CEBRID - Centro Brasileiro de Informação sobre Drogas Psicotrópicas: UNIFESP - Universidade Federal de São Paulo; 2006. 42 p.

7. Ferreira ACZ, Capistrano FC, Souza EB, Borba LO, Kalinke LP, Maftum MA. Motivações de dependentes químicos para o tratamento: percepção de familiares. Revista Brasileira de enfermagem. 2015;68(3):474-481.

8. Barreto AP. Terapia comunitária: passo a passo. $4^{\mathrm{a}} \mathrm{ed}$. Revista ampliada. Fortaleza: LCR, 2008. 407 p.

9. Ferreira Filha MO, Dias MD, Andrade FB, Lima ÉAR, Ribeiro FF, Silva MSS. A Terapia Comunitária como estratégia de promoção à saúde mental: o caminho para o empoderamento. Rev Eletrôn Enferm. 2009;11(4):964-70.

10. Brito LDL. Terapia Comunitária Integrativa como cuidado complementar na reabilitação de dependentes químicos [Monografia]. Curso de Enfermagem: Universidade Federal de Mato Grosso, Campus Universitário do Araguaia, Barra do Garças - MT; 2016. 73 p.

11. Bardin L. Análise de conteúdo. São Paulo: Edições 70; 2011. 
12. Possa T, Durman S. Processo de ressocialização de usuários de substâncias lícitas e ilícitas. SMAD Rev Eletrôn Saúde Mental Álcool Drogas. 2007;3(2):1-14.

13. Ferro LRM, Meneses-Gaya C. Resiliência como fator protetor no consumo de drogas entre universitários. Saúde e Pesquisa. 2015;8:139-49.

14. Silva JL. Terapia de rede para adictos: programa de tratamento e prevenção para dependentes de drogas em comunidades terapêuticas [Tese]. Faculdade de Saúde Pública da Universidade de São Paulo; 2011. 222 p.

15. Tissot CL. A influência da familia sobre a adesão ao tratamento do dependente químico: um estado piloto sobre a emoção expressa [Dissertação]. Faculdade de Medicina da Universidade de São Paulo; 2006. 113 p.

16. Kessler F, Faller S, Formigoni MLOS, Cruz MS, Brasiliano S, Stolf AR, et al. Avaliação multidimensional do usuário de drogas e a Escala de Gravidade de Dependência. Rev Psiquiatria Rio Grande do Sul. 2010;32(2):48-56.

17. Rezende MM, Pelicia B. Representação da Recaída em dependentes de crack. SMAD, Rev Eletrônica Saúde Mental Álcool Drogas. 2013;9(2):76-81.

18. Ferreira Filha MO, Lazarte R, Dias MD. Terapia Comunitária Integrativa uma construção coletiva do conhecimento. In: Carício MR, Dias MD, Franco TB, Ferreira MO Filha. Terapia Comunitária: um encontro que transforma o jeito de ver e conduzir a vida. João PessoaPB: Ed. Universitária da UFPB; 2013. p. 132-58.

19. Ferreira MO Filha, Lazarte R, Dias MD. Terapia Comunitária Integrativa uma construção coletiva do conhecimento. In: Morais FLSL, Dias MD, Ferreira Filha MO. Rodas de terapia comunitária: espaços de mudanças para profissionais da estratégia de saúde da família. -João Pessoa-PB: Ed. Universitária da UFPB; 2013. p. 159-87.

20. Rocha IA, Sá ANP, Braga LAV, Ferreira Filha MO, Dias MD. Terapia comunitária integrativa: situações de sofrimento emocional e estratégias de enfrentamento apresentadas por usuários. Rev Gaúch Enferm. 2013;34(2):155-62.

21. Melo OS, Ribeiro LRR, Costa ALRC, Urel DR. Repercussões da terapia comunitária integrativa nas pessoas doentes renais durante sessão de hemodiálise. Rev Pesqui cuidado é fundamental. 2015;7(2):2200-14.

22. Rangel CT, Miranda FAN, Oliveira KKD. A terapia comunitária integrativa e a enfermagem: o fenômeno e seus contextos. Rev Pesqui cuidado é fundamental. 2016;8(1):3770-9.

23. Braga LAV, Dias MD, Ferreira Filha MO, Moraes MN, Araruna MHM, Rocha IA. Terapia Comunitária e resiliência: história de mulheres. Revista de pesquisa: cuidado é fundamental. 2013;5(1):3453-71.
24. Carvalho MAP, Dias MD, Miranda FAN, Ferreira Filha MO. Contribuições da terapia comunitária integrativa para usuários dos Centros de Atenção Psicossocial (CAPS): do isolamento à sociabilidade libertadora. Cad Saúde Pública. 2013;29(10):2028-038.

25. Ribeiro FML, Minayo MCS. As Comunidades Terapêuticas religiosas na recuperação de dependentes de drogas: o caso de Manguinhos, RJ, Brasil. Interface: comunicação saúde educação. 2015;19(54):515-26.

26. Felipe OAB, Carvalho AMP, Andrade CUB. Espiritualidade e religião como protetores ao uso de drogas em adolescente. SMAD, Rev. Eletrônica Saúde Mental Álcool Drogas. 2015;11(1):49-58. 